\title{
Research Article \\ Prevention of Influenza Pandemic by Multiple Control Strategies
}

\author{
Roman Ullah, ${ }^{1}$ Gul Zaman, ${ }^{2}$ and Saeed Islam ${ }^{1}$ \\ ${ }^{1}$ Department of Mathematics, Abdul Wali Khan University, Mardan, \\ Khyber Pakhtunkhwa 23200, Pakistan \\ ${ }^{2}$ Department of Mathematics, University of Malakand, Chakdara, Lower Dir, \\ Khyber Pakhtunkhwa 23101, Pakistan \\ Correspondence should be addressed to Roman Ullah, romanullah@yahoo.com
}

Received 4 October 2012; Accepted 25 November 2012

Academic Editor: Junjie Wei

Copyright (C) 2012 Roman Ullah et al. This is an open access article distributed under the Creative Commons Attribution License, which permits unrestricted use, distribution, and reproduction in any medium, provided the original work is properly cited.

\begin{abstract}
We present the prevention of influenza pandemic by using multiple control functions. First, we adjust the control functions in the pandemic model, then we show the existence of the optimal control problem, and, by using both analytical and numerical techniques, we investigate costeffective control effects for the prevention of transmission of disease. To do this, we use four control functions, the first one for increasing the effect of vaccination, the second one for the strategies to isolate infected individuals, and the last two for the antiviral treatment to control clinically infectious and hospitalization cases, respectively. We completely characterized the optimal control and compute the numerical solution of the optimality system by using an iterative method.
\end{abstract}

\section{Introduction}

Influenza is a seasonal viral disease caused by influenza A virus (H1N1) which spreads rapidly, and it costs the society a significant amount in terms of morbidity and mortality with a typical flu epidemic. It is estimated that more than 30 million people have been killed by human influenza, having a considerable impact on public health. The threat of recent avian influenza epidemics is also causing a widespread public concern [1]. The direct contact with poultry increased the number of avian flu cases in humans. The urgency to develop pandemic preparedness worldwide is prompted in many regions of the world. The international organizations are trying to implement a strategy to delay or minimize the impact of onset of a pandemic [2]. Influenza viruses have historically been a cause of large number of mortality [3]. It is estimated by WHO that from 5 to $15 \%$ of the world population is effected each year by the seasonal influenza, causing from 250,000 to 500,000 deaths each 
year $[2,4,5]$. Due to the high rate of mortality, the preventive strategies attracted the attention of the researchers [3]. After the announcement by WHO that the infectious diseases are spreading faster than any time in the history, the pandemic influenza poses a severe threat to public health. Vaccines are the leading recommendations to prevent infection and to control the spread of the disease. Despite the public health vaccination programs and the availability of the vaccination influenza inflicts a large number of mortality and remains a major problem for public health because the protection conferred by current vaccines is dependent on the immune status of the individual [6]. To control the spread of influenza, a strategic use of partially effective vaccines is of great public health interest.

In the last decade, various studies of the influenza pandemic have been carried out. Alexander et al. [6] explore the impact of immunization with a partially effective vaccine via a mathematical model for the transmission dynamics of influenza. Therein they discussed two cases; the first one is the case in which the population does not admit the inflow of new infected individual and in second case the population does admit it. In their work, the rate of vaccination is based on the rate of contact between the susceptible and infected individuals leading to the infection and the duration of infectiousness. The aid of a partially effective vaccine is very crucial for controlling the rapid spread of influenza. In 2006, Iwami et al. [7] proposed a mathematical model for the spread of bird flu from the bird population to the human population. They discussed that, to minimize the spread of the disease in human population, someone must take the measures for the infected human with bird flu to quarantine when mutant bird flu has already occurred. In order to evaluate the pandemic flu preparedness plans of the Netherland, United Kingdom and United States., Nuño et al. [8] analyzed a more complex mathematical model. Their results showed that antiviral and vaccines give the most optimal results, but, due to the lack of medical facilities and limited antiviral stockpiles, the developing countries must emphasize their use therapeutically.

Vaccination is the primary method for preventing influenza and its severe complications. The vaccination might prevent hospitalization and can reduce influenza-related respiratory illness. The level of vaccination increased substantially in the last two decades but still further improvements in vaccination levels are needed, especially among the aged people. Although influenza vaccination remains the cornerstone for the control of influenza, the production of vaccines particularly the new H1N1 vaccine that the world is eager, some would say desperate to buy, raises concerns at multiple levels [9]. Antiviral medications can also play a significant role in controlling the spread of influenza as the antiviral drugs are thought to shorten duration of the infectious period, and to reduce transmission of the virus. The supply of antiviral and demand does not meet in the developing countries. The people in poor nations are unable to get the timely access to minimally adequate vaccine or drug stockpiles.

In this paper, we focus to identify the optimal control strategies that minimize the impact of influenza by minimizing the vaccine wanning, the judicious use of drug supply, and isolating the clinically infectious patients. Vaccination coverage can be increased by administering vaccines to individuals during hospitalization or routine health care visits as well as pharmacies, grocery stores, work places, and other locations in the community before the influenza season. The risk of the spread of influenza and subsequent influenzarelated complications can also be reduced by vaccinating the health care workers and other persons in closed contact with persons at increased risk of severe influenza. The clinically infectious patients can be isolated by reasonably effective ways to reduce the transmission of influenza, like to educate them to cover their sneeze and cough, not to spit openly, to avoid the closed contacts with others, and sanitizing the rooms or equipments occupied by 
the patients by using quaternary ammonium compounds and bleach. We can get the most effective results if we use the vaccination, treatment, and isolation of the clinically infected patients concurrently. For developing countries this model is very suitable as only a few people can get vaccinated, some of the people may have access to the treatment, and in some places the disease can be controlled by isolation.

Our paper is organized as follows. In Section 2, we introduced the control model of the disease. In Section 3, we checked the existence of the control problem. In Section 4, the numerical solution of the optimality system is computed by using semi-implicit finite difference method. A short conclusion is given in Section 5.

\section{Influenza Model with Controls}

The model in this section presents the optimal control problem for the transmission dynamics of influenza. Our main aim is to show that it is possible to implement the time-dependent anti-influenza control techniques while minimizing the cost of such measures. In our optimal control problem, we introduce four control functions $u_{1}, u_{2}, u_{3}$, and $u_{4}$. The control $u_{1}$ represents the successful efforts to minimize the immunity wanning by administering vaccine to persons during hospitalization or routine health care visits as well as pharmacies, workplaces, or other locations in the community before the season of influenza. $u_{2}$ represents the isolation of the clinically infected patients by covering coughs, sneezing, not to spit openly, and avoiding the closed contacts with others. $u_{3}$ and $u_{4}$ represent the fraction of clinically infected cases treated with antiviral per unit of time and the fraction of individuals getting antiviral treatments at hospitals per unit of time, respectively. Note that the controls are fully effective when $u_{i}=1$ for $i=1,2,3,4$, while there is no control if $u_{i}=0$. We divide the total population into six distinct subclasses which are susceptible class $S$, vaccinated class $V$, exposed class $E$, clinically ill and infectious class $I$, treated class $T$, and recovered class $R$. Taking into account the assumptions above, the dynamics of the control problem is given by

$$
\begin{aligned}
& \frac{d S}{d t}=\Lambda+\left(1-u_{1}\right) k V-\alpha_{1} S E-\left(1-u_{2}\right) \alpha_{2} S I-(\phi+\mu) S+r R, \\
& \frac{d V}{d t}=\phi S-\left(1-u_{1}\right) k V-(1-\sigma) \alpha_{1} V E-(1-\sigma) \alpha_{2} V I-\mu V, \\
& \frac{d E}{d t}=\alpha_{1} S E+\left(1-u_{2}\right) \alpha_{2} S I+(1-\sigma) V\left[\alpha_{1} E+\alpha_{2} I\right]-\left(\alpha_{3}+\mu\right) E, \\
& \frac{d I}{d t}=\alpha_{3} E-\left(\mu+w+v+\varepsilon+u_{3}\right) I, \\
& \frac{d T}{d t}=w I-\left[\beta+\mu+v(1-\theta)+u_{4}\right] T, \\
& \frac{d R}{d t}=\varepsilon I+\beta T-(r+\mu) R+u_{3} I+u_{4} T,
\end{aligned}
$$

with the initial conditions

$$
S(0) \geq 0, \quad V(0) \geq 0, \quad E(0) \geq 0, \quad I(0) \geq 0, \quad T(0) \geq 0, \quad R(0) \geq 0 .
$$


The population is recruited at a constant birth rate $\Lambda . k$ is the rate at which the vaccinationbased immunity wanes, and $\alpha_{1}$ and $\alpha_{2}$ are effective contact rates between the susceptible individuals and the infected and exposed individuals, respectively. $\phi$ is the rate at which susceptible individuals are vaccinated, $\sigma$ represents vaccine efficacy, $\sigma \in[0,1]$, the vaccine is imperfect if $0<\sigma<1$, the vaccine is perfect if $\sigma=1$ and is useless if $\sigma=0, w$ is the rate at which individuals transfer from exposed class to infected class, $\mu$ is natural death rate, and $v$ is disease-induced death rate. $\theta$ is effectiveness of the treatment as a reduction factor in disease-induced death of infected individuals $(0<\theta \leq 1), r_{1}$ is the rate of immunity loss, $r_{2}$ represents treatment rate, $r_{3}$ is the natural recovery rate of infected individuals, and $r_{4}$ is recovery rate due to treatment.

The objective of our work is to minimize infected and hospitalized population and the cost of implementing the control by using possible minimal control variables $u_{i}(t)$ for $i=1,2,3,4$. We use the Lebesgue measurable control and define our objective functional as

$$
J\left(u_{1}, u_{2}, u_{3}, u_{4}\right)=\int_{0}^{t_{\text {end }}}\left(A_{1} I+A_{2} T+\frac{1}{2}\left(C_{1} u_{1}^{2}+C_{2} u_{2}^{2}+C_{3} u_{3}^{2}+C_{4} u_{4}^{2}\right)\right) d t
$$

The quantities $A_{1}, A_{2}$, and $C_{i}$, where $i=1, \ldots, 4$, represent a measure of the relative cost of the interventions over $\left[0, t_{\text {end }}\right]$. The objective of the optimal control problem is to seek optimal control functions $\left(u_{1}^{*}(t), u_{2}^{*}(t), u_{3}^{*}(t), u_{4}^{*}(t)\right)$ such that

$$
J\left(u_{1}^{*}, u_{2}^{*}, u_{3}^{*}, u_{4}^{*}\right)=\min _{\left(u_{1}, u_{2}, u_{3}, u_{4}\right) \in U}\left\{J\left(u_{1}, u_{2}, u_{3}, u_{4}\right) \mid\left(u_{1}, u_{2}, u_{3}, u_{4}\right) \in U\right\}
$$

where the control set is defined as

$$
\begin{gathered}
U=\left\{u=\left(u_{1}, u_{2}, u_{3}, u_{4}\right) \mid u_{i} \text { is Lebesgue measurable on }[0,1],\right. \\
\left.0 \leq u_{i}(t) \leq 1, t \in\left[0, t_{\text {end }}\right], \text { for } i=1, \ldots, 4\right\}
\end{gathered}
$$

subject to the system (2.1) and for appropriate initial conditions. Pontryagin's Maximum Principle is used to solve this optimal control problem and the derivation of the necessary conditions. First, we prove the existence of the control problem (2.1) and then derive the optimality system.

\section{Existence of Control Problem}

In this section, we consider the control system (2.1) with initial conditions (2.2) to show the existence of the control problem. Note that, for the bounded Lebesgue measurable controls and nonnegative initial conditions, nonnegative bounded solutions to the state system exist [10]. Let us go back to the optimal control problem (2.1)-(2.3). In order to find an optimal solution, first we should find the Lagrangian and Hamiltonian for the optimal control problem. The minimal value of the Lagrangian is given by

$$
L=A_{1} I+A_{2} T+\frac{1}{2}\left(C_{1} u_{1}^{2}+C_{2} u_{2}^{2}+C_{3} u_{3}^{2}+C_{4} u_{4}^{2}\right)
$$


We define the Hamiltonian $H$ for the control problem, where $\lambda_{i}, i=1,2, \ldots, 6$ are the adjoint variables:

$$
\begin{aligned}
H= & A_{1} I+A_{2} T+\frac{1}{2}\left(C_{1} u_{1}^{2}+C_{2} u_{2}^{2}+C_{3} u_{3}^{2}+C_{4} u_{4}^{2}\right) \\
& +\lambda_{1}\left[\Lambda+\left(1-u_{1}\right) k V-\alpha_{1} S E-\left(1-u_{2}\right) \alpha_{2} S I-(\phi+\mu) S+r R\right] \\
& +\lambda_{2}\left[\phi S-\left(1-u_{1}\right) k V-(1-\sigma) \alpha_{1} V E-(1-\sigma) \alpha_{2} V I-\mu V\right] \\
& +\lambda_{3}\left[\alpha_{1} S E+\left(1-u_{2}\right) \alpha_{2} S I+(1-\sigma) \alpha_{1} V E+(1-\sigma) \alpha_{2} V I\right. \\
& \left.\quad-\left(\alpha_{3}+\mu\right) E\right]+\lambda_{4}\left[\alpha_{3} E-\left(\mu+w+v+\varepsilon+u_{3}\right) I\right] \\
& +\lambda_{5}\left[w I-\left[\beta+\mu+v(1-\theta)+u_{4}\right] T\right] \\
& +\lambda_{6}\left[\varepsilon I+\beta T-(r+\mu) R+u_{3} I+u_{4} T\right] .
\end{aligned}
$$

For the existence of our control system (2.1), we state and prove the following theorem.

Theorem 3.1. There exists an optimal control $u^{*}=\left(u_{1}^{*}, u_{2}^{*}, u_{3}^{*}, u_{4}^{*}\right) \in U$ such that

$$
J\left(u_{1}^{*}, u_{2}^{*}, u_{3}^{*}, u_{4}^{*}\right)=\min _{\left(u_{1}, u_{2}, u_{3}, u_{4}\right) \in U} J\left(u_{1}, u_{2}, u_{3}, u_{4}\right)
$$

subject to the control system (2.1) with the initial conditions (2.2).

Proof. To prove the existence of an optimal control we use the result in [11-13]. Note that the control and the state variables are nonnegative values. In this minimizing problem, the necessary convexity of the objective functional in $u_{1}, u_{2}, u_{3}$, and $u_{4}$ is satisfied. The set of all the control variables $\left(u_{1}, u_{2}, u_{3}, u_{4}\right) \in U$ is also convex and closed by definition. The optimal system is bounded which determines the compactness needed for the existence of an optimal control. In addition the integrand in the functional (2.3), $A_{1} I+A_{2} T+1 / 2\left(C_{1} u_{1}^{2}+C_{2} u_{2}^{2}+C_{3} u_{3}^{2}+\right.$ $C_{4} u_{4}^{2}$ ), is convex on the control set $U$. Also we can see that there exist a constant $\rho>1$ and positive numbers $\omega_{1}, \omega_{2}$ such that

$$
J\left(u_{1}, u_{2}, u_{3}, u_{4}\right) \geq \omega_{1}\left(\left|u_{1}\right|^{2}+\left|u_{2}\right|^{2}+\left|u_{3}\right|^{2}+\left|u_{4}\right|^{2}\right)^{\rho / 2}-\omega_{2}
$$

because the state variables are bounded, which completes the existence of an optimal control. 
In order to derive the necessary conditions, we use Pontryagin's maximum principle [14] as follows. If $(x, u)$ is an optimal solution of an optimal control problem, then there exists a nontrivial vector function $\lambda=\left(\lambda_{1}, \lambda_{2}, \ldots, \lambda_{n}\right)$ satisfying the following equations:

$$
\begin{aligned}
& \frac{d x}{d t}=\frac{\partial H(t, x, u, \lambda)}{\partial \lambda}, \\
& 0=\frac{\partial H(t, x, u, \lambda)}{\partial u}, \\
& \frac{d \lambda}{d t}=\frac{\partial H(t, x, u, \lambda)}{\partial x} .
\end{aligned}
$$

We now derive the necessary conditions that optimal control functions and corresponding states must satisfy. In the following theorem, we present the adjoint system and control characterization.

Theorem 3.2. Let $S^{*}, V^{*}, E^{*}, I^{*}, T^{*}$, and $R^{*}$ be optimal state solutions with associated optimal control variables $\left(u_{1}^{*}, u_{2}^{*}, u_{3}^{*}, u_{4}^{*}\right)$ for the optimal control problem (2.1)-(2.3). Then there exist adjoint variables $\lambda_{i}$, for $i=1,2, \ldots, 6$ satisfying

$$
\begin{aligned}
& \frac{d \lambda_{1}}{d t}=\left(\lambda_{1}-\lambda_{3}\right)\left(\alpha_{1} E+\left(1-u_{2}\right) \alpha_{2} I\right)+\left(\lambda_{1}-\lambda_{2}\right) \phi+\lambda_{1} \mu, \\
& \frac{d \lambda_{2}}{d t}=\left(1-u_{1}\right)\left(\lambda_{2}-\lambda_{1}\right) k+\left(\lambda_{2}-\lambda_{3}\right)(1-\sigma)\left(\alpha_{1} E+\alpha_{2} I\right)+\lambda_{2} \mu, \\
& \frac{d \lambda_{3}}{d t}=\left(\lambda_{1}-\lambda_{3}\right) \alpha_{1} S+\left(\lambda_{2}-\lambda_{3}\right)(1-\sigma) \alpha_{1} V+\left(\lambda_{3}-\lambda_{4}\right) \alpha_{3}+\lambda_{3} \mu, \\
& \frac{d \lambda_{4}}{d t}=\left(\lambda_{1}-\lambda_{3}\right)\left(1-u_{2}\right) \alpha_{2} S+\left(\lambda_{2}-\lambda_{3}\right)(1-\sigma) \alpha_{2} V \\
& \quad+\left(\lambda_{4}-\lambda_{6}\right)\left(\varepsilon+u_{3}\right)+\left(\lambda_{4}-\lambda_{5}\right) w+\lambda_{4}(v+\mu)-A_{1}, \\
& \frac{d \lambda_{5}}{d t}=\left(\lambda_{5}-\lambda_{6}\right)\left(\beta+u_{4}\right)+\lambda_{5}(\mu+v(1-\theta))-A_{2}, \\
& \frac{d \lambda_{6}}{d t}=\left(\lambda_{6}-\lambda_{1}\right) r+\lambda_{6} \mu
\end{aligned}
$$

with transversality conditions

$$
\lambda_{i}\left(t_{\text {end }}\right)=0, \quad i=1, \ldots, 6
$$

Furthermore the control functions $u_{1}^{*}, u_{2}^{*}, u_{3}^{*}$, and $u_{4}^{*}$ are given by

$$
u_{1}^{*}=\max \left\{\min \left\{\frac{\left(\lambda_{1}-\lambda_{2}\right) k V^{*}}{c_{1}}, 1\right\}, 0\right\},
$$


Journal of Applied Mathematics

$$
\begin{aligned}
& u_{2}^{*}=\max \left\{\min \left\{\frac{\left(\lambda_{3}-\lambda_{1}\right) \alpha_{2} S^{*} I^{*}}{c_{2}}, 1\right\}, 0\right\}, \\
& u_{3}^{*}=\max \left\{\min \left\{\frac{\left(\lambda_{4}-\lambda_{6}\right) I^{*}}{c_{3}}, 1\right\}, 0\right\}, \\
& u_{4}^{*}=\max \left\{\min \left\{\frac{\left(\lambda_{5}-\lambda_{6}\right) T^{*}}{c_{4}}, 1\right\}, 0\right\} .
\end{aligned}
$$

Proof. To determine the adjoint equations and the transversality conditions, we use the Hamiltonian $H$ in (3.2). The adjoint system results from the Pontryagin's Maximum Principle [14]:

$$
\frac{d \lambda_{1}}{d t}=-\frac{\partial H}{\partial S}, \quad \frac{d \lambda_{2}}{d t}=-\frac{\partial H}{\partial V}, \quad \frac{d \lambda_{3}}{d t}=-\frac{\partial H}{\partial E}, \quad \frac{d \lambda_{4}}{d t}=-\frac{\partial H}{\partial I}, \quad \frac{d \lambda_{5}}{d t}=-\frac{\partial H}{\partial T}, \quad \frac{d \lambda_{6}}{d t}=-\frac{\partial H}{\partial R}
$$

with $\lambda_{i}\left(t_{\text {end }}\right)=0, i=1,2, \ldots, 6$.

To get the characterization of the optimal control given by (3.8), solving the equations,

$$
\frac{\partial H}{\partial u_{1}}=0, \quad \frac{\partial H}{\partial u_{2}}=0, \quad \frac{\partial H}{\partial u_{3}}=0, \quad \frac{\partial H}{\partial u_{4}}=0,
$$

on the interior of the control set and setting the property of the control space $U$, we can derive the desired characterization (3.8).

\section{Numerical Results and Discussion}

In this section, we present a semi-implicit finite difference method by discretizing the interval $\left[t_{0}, t_{f}\right]$ at the points $t_{i}=t_{0}+i l,(i=0,1, \ldots, n)$, where $l$ represents the time step such that $t_{n}=t_{f}$. We define the state and adjoint variables $S, V, E, I, T, R, \lambda_{1}, \lambda_{2}, \lambda_{3}, \lambda_{4}, \lambda_{5}, \lambda_{6}$ and the controls $u_{1}, u_{2}, u_{3}, u_{4}$ in terms of nodal points $S^{i}, V^{i}, E^{i}, I^{i}, T^{i}, R^{i}, \lambda_{1}^{i}, \lambda_{2}^{i}, \lambda_{3}^{i}, \lambda_{4}^{i}, \lambda_{5}^{i}, \lambda_{6}^{i}, u_{1}^{i}, u_{2}^{i}, u_{3}^{i}$, and $u_{4}^{i}$. By combination of forward and backward difference approximation, the method developed by [15], to adopt it in our case is as following:

$$
\begin{aligned}
\frac{S^{i+1}+S^{i}}{l}= & \Lambda+\left(1-u_{1}^{i}\right) k V^{i}-\alpha_{1} S^{i+1} E^{i}-\left(1-u_{2}^{i}\right) \alpha_{2} S^{i+1} I^{i}-(\phi+\mu) S^{i+1}+r R^{i}, \\
\frac{V^{i+1}+V^{i}}{l}= & \phi S^{i+1}-\left(1-u_{1}^{i}\right) k V^{i+1}-(1-\sigma) \alpha_{1} V^{i+1} E^{i}-(1-\sigma) \alpha_{2} V^{i+1} I^{i}-\mu V^{i+1}, \\
\frac{E^{i+1}+E^{i}}{l}= & \alpha_{1} S^{i+1} E^{i}+\left(1-u_{2}^{i}\right) \alpha_{2} S^{i+1} I^{i}+(1-\sigma) \alpha_{1} V^{i+1} E^{i+1} \\
& +(1-\sigma) \alpha_{2} V^{i+1} I^{i}-\left(\alpha_{3}+\mu\right) E^{i+1},
\end{aligned}
$$




$$
\begin{aligned}
& \frac{I^{i+1}+I^{i}}{l}=\alpha_{3} E^{i+1}-\left(\mu+w+v+\varepsilon+u_{3}^{i}\right) I^{i+1}, \\
& \frac{T^{i+1}+T^{i}}{l}=w I^{i+1}-\left[\beta+\mu+v(1-\theta)+u_{4}^{i}\right] T^{i+1}, \\
& \frac{R^{i+1}+R^{i}}{l}=\varepsilon I^{i+1}+\beta T^{i+1}-(r+\mu) R^{i+1}+u_{3}^{i} I^{i+1}+u_{4}^{i} T^{i+1} .
\end{aligned}
$$

To approximate the time derivative of the adjoint variables by the first-ordered backward difference, we use the appropriate scheme as follows:

$$
\begin{aligned}
& \frac{\lambda_{1}^{n-i}-\lambda_{1}^{n-i-1}}{l}=\left(\lambda_{1}^{n-i-1}-\lambda_{3}^{n-i}\right)\left[\alpha_{1} E^{i+1}+\alpha_{2}\left(1-u_{2}^{i}\right) I^{i+1}\right]+\left(\lambda_{1}^{n-i-1}-\lambda_{2}^{n-i}\right) \phi+\lambda_{1}^{n-i-1} \mu, \\
& \frac{\lambda_{2}^{n-i}-\lambda_{2}^{n-i-1}}{l}=\left(\lambda_{2}^{n-i-1}-\lambda_{1}^{n-i-1}\right)\left(1-u_{1}^{i}\right) k+\left(\lambda_{2}^{n-i-1}-\lambda_{3}^{n-i}\right)(1-\sigma)\left[\alpha_{1} E^{i+1}+\alpha_{2} I^{i+1}\right]+\lambda_{2}^{n-i-1} \mu, \\
& \frac{\lambda_{3}^{n-i}-\lambda_{3}^{n-i-1}}{l}=\left(\lambda_{1}^{n-i-1}-\lambda_{3}^{n-i-1}\right) \alpha_{1} S^{i+1}+\left(\lambda_{2}^{n-i-1}-\lambda_{3}^{n-i-1}\right)(1-\sigma) \alpha_{1} V^{i+1} \\
&+\lambda_{3}^{n-i-1} \mu+\left(\lambda_{3}^{n-i-1}-\lambda_{4}^{n-i}\right) \alpha_{3} \\
&+\left(\lambda_{4}^{n-i-1}+\lambda_{5}^{n-i}\right) w+\left(\lambda_{4}^{n-i-1}+\lambda_{6}^{n-i}\right)\left(\varepsilon+u_{3}^{i}\right)+\lambda_{4}^{n-i-1}(v+\mu)-A_{1}, \\
& \frac{\lambda_{4}^{n-i}-\lambda_{4}^{n-i-1}=}{l}\left(\lambda_{1}^{n-i-1}-\lambda_{3}^{n-i-1}\right)\left(1-u_{2}^{i}\right) \alpha_{2} S^{i+1}+\left(\lambda_{2}^{n-i-1}-\lambda_{3}^{n-i-1}\right)(1-\sigma) \alpha_{2} V^{i+1} \\
& \frac{\lambda_{5}^{n-i}-\lambda_{5}^{n-i-1}=}{l}\left(\lambda_{5}^{n-i-1}-\lambda_{6}^{n-i}\right)\left(\beta+u_{4}^{i}\right)+\lambda_{5}^{n-i-1}[\mu+v(1-\theta)]-A_{2}, \\
& \frac{\lambda_{6}^{n-i}-\lambda_{6}^{n-i-1}=}{l}\left(\lambda_{6}^{n-i-1}-\lambda_{1}^{n-i-1}\right) r+\lambda_{6}^{n-i-1} \mu .
\end{aligned}
$$

The algorithm that describes the approximation method for obtaining the optimal control is as follows.

\section{Algorithm 4.1}

Step 1. $S(0)=S_{0}, V(0)=V_{0}, E(0)=E_{0}, I(0)=I_{0}, T(0)=T_{0}, R(0)=R_{0}, u_{1}(0)=u_{2}(0)=u_{3}(0)=$ $u_{4}(0)=0, \lambda_{i}\left(t_{f}\right)=0, i=1, \ldots, 6$.

Step 2. For $i=1, \ldots, n-1$, do the following:

$$
\begin{aligned}
S^{i+1} & =\frac{S^{i}+l\left[\Lambda+\left(1-u_{1}^{i}\right) k V^{i}+r_{1} R^{i}\right]}{1+l\left[\phi+\mu+\alpha_{1} E^{i}+\left(1-u_{2}^{i}\right) \alpha_{2} I^{i}\right]}, \\
V^{i+1} & =\frac{V^{i}+l \phi S^{i+1}}{1+l\left[\left(1-u_{1}^{i}\right) k+(1-\sigma) \alpha_{1} E^{i}+(1-\sigma) \alpha_{2} I^{i}+\mu\right]},
\end{aligned}
$$




$$
\begin{aligned}
& E^{i+1}=\frac{E^{i}+l\left[\left(1-u_{2}^{i}\right) \alpha_{2} S^{i+1} I^{i}+(1-\sigma) \alpha_{2} V^{i+1} I^{i}\right]}{1+l\left[\alpha_{3}+\mu+(1-\sigma) \alpha_{1} V^{i+1}-\alpha_{1} S^{i+1}\right]}, \\
& I^{i+1}=\frac{I^{i}+l \alpha_{3} E^{i+1}}{1+l\left[\mu+w+v+\varepsilon+u_{3}^{i}\right]} \\
& T^{i+1}=\frac{T^{i}+l w I^{i+1}}{1+l\left[\beta+\mu+v(1-\theta)+u_{4}^{i}\right]}, \\
& R^{i+1}=\frac{R^{i}+l\left[\varepsilon I^{i+1}+\beta T^{i+1}+u_{3}^{i} I^{i+1}+u_{4}^{i} T^{i+1}\right]}{1+l[r+\mu]}, \\
& \lambda_{1}^{n-i-1}=\frac{\lambda_{1}^{n-i}+l\left[\alpha_{1} E^{i+1} \lambda_{3}^{n-i}+\left(1-u_{2}^{i}\right) \alpha_{2} I^{i+1} \lambda_{3}^{n-i}+\phi \lambda_{2}^{n-i}\right]}{1+l\left[\phi+\mu+\alpha_{1} E^{i+1}+\left(1-u_{2}^{i}\right) \alpha_{2} I^{i+1}\right]}, \\
& \lambda_{2}^{n-i-1}=\frac{\lambda_{2}^{n-i}+l\left[\left(1-u_{1}^{i}\right) k \lambda_{1}^{n-i-1}+(1-\sigma)\left[\alpha_{1} E^{i+1}+\alpha_{2} I^{i+1}\right] \lambda_{3}^{n-i}\right]}{1+l\left[1+\mu+\left(1-u_{1}^{i}\right) k+(1-\sigma)\left(\alpha_{1} E^{i+1}+\alpha_{2} I^{i+1}\right)\right]}, \\
& \lambda_{3}^{n-i-1}=\frac{\lambda_{3}^{n-i}+l\left[\lambda_{1}^{n-i-1} \alpha_{1} S^{i+1}+\lambda_{2}^{n-i-1}(1-\sigma) \alpha_{1} V^{i+1}-\lambda_{4}^{n-i} \alpha_{3}\right]}{1+l\left[\alpha_{1} S^{i+1}+(1-\sigma) \alpha_{1} V^{i+1}-\alpha_{3}-\mu\right]}, \\
& \lambda_{4}^{n-i-1}=\left(\lambda_{4}^{n-i}+l\left[\left(\lambda_{3}^{n-i-1}-\lambda_{1}^{n-i-1}\right)\left(1-u_{2}^{i}\right) \alpha_{2} S^{i+1}+\left(\lambda_{2}^{n-i-1}-\lambda_{3}^{n-\mathrm{i}-1}\right)(1-\sigma) \alpha_{2} V^{i+1}\right.\right. \\
& \left.\left.+\lambda_{6}^{n-i}\left(\varepsilon+u_{3}^{i}\right)+\lambda_{5}^{n-i} w-\lambda_{4}^{n-i} \mu+A_{1}\right]\right)\left(1+l\left[\varepsilon+w+v+\mu+u_{3}^{i}\right]\right)^{-1}, \\
& \lambda_{5}^{n-i-1}=\frac{\lambda_{5}^{n-i}+l\left[\lambda_{6}^{n-i}\left(\beta+u_{4}^{i}\right)+A_{2}\right]}{1+l\left[\beta+\mu+v(1-\theta)+u_{4}^{i}\right]}, \\
& \lambda_{6}^{n-i-1}=\frac{\lambda_{6}^{n-i}+l r \lambda_{1}^{n-i-1}}{1+l[r+\mu]}, \\
& u_{1}^{i+1}=\min \left\{1, \max \left\{\frac{\left(\lambda_{1}^{n-i-1}-\lambda_{2}^{n-i-1}\right) k V^{i+1}}{c_{1}}, 0\right\}\right\}, \\
& u_{2}^{i+1}=\min \left\{1, \max \left\{\frac{\left(\lambda_{3}^{n-i-1}-\lambda_{1}^{n-i-1}\right) \alpha_{2} S^{i+1} I^{i+1}}{c_{2}}, 0\right\}\right\} \text {, } \\
& u_{3}^{i+1}=\min \left\{1, \max \left\{\frac{\left(\lambda_{4}^{n-i-1}-\lambda_{6}^{n-i-1}\right) I^{i+1}}{c_{3}}, 0\right\}\right\} \text {, } \\
& u_{4}^{i+1}=\min \left\{1, \max \left\{\frac{\left(\lambda_{5}^{n-i-1}-\lambda_{6}^{n-i-1}\right) T^{i+1}}{c_{4}}, 0\right\}\right\}
\end{aligned}
$$

end for. 


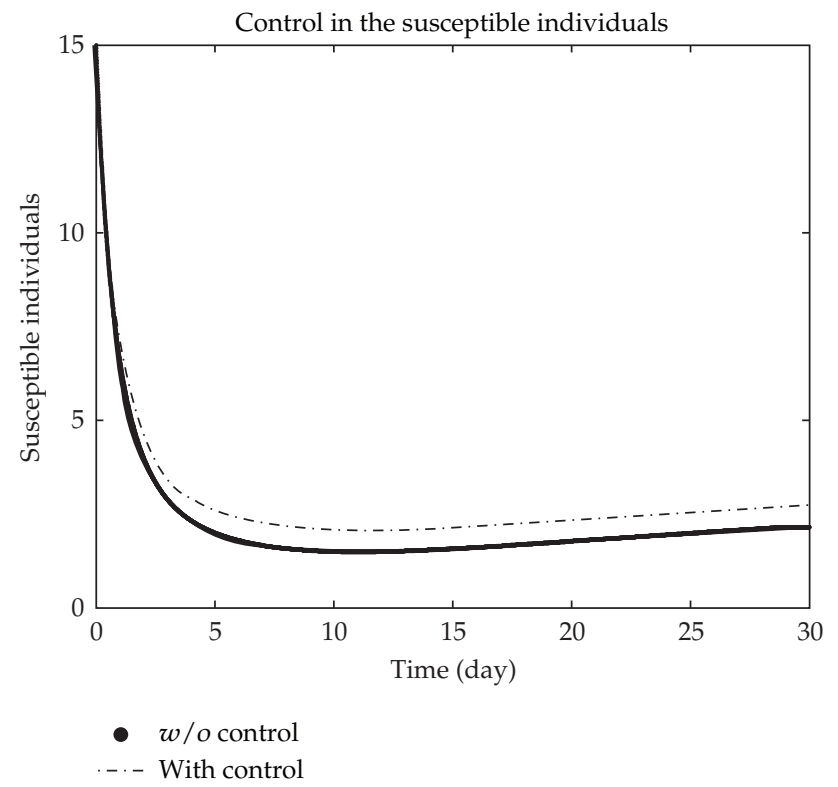

Figure 1: The plot represents the population of susceptible class with and without control.

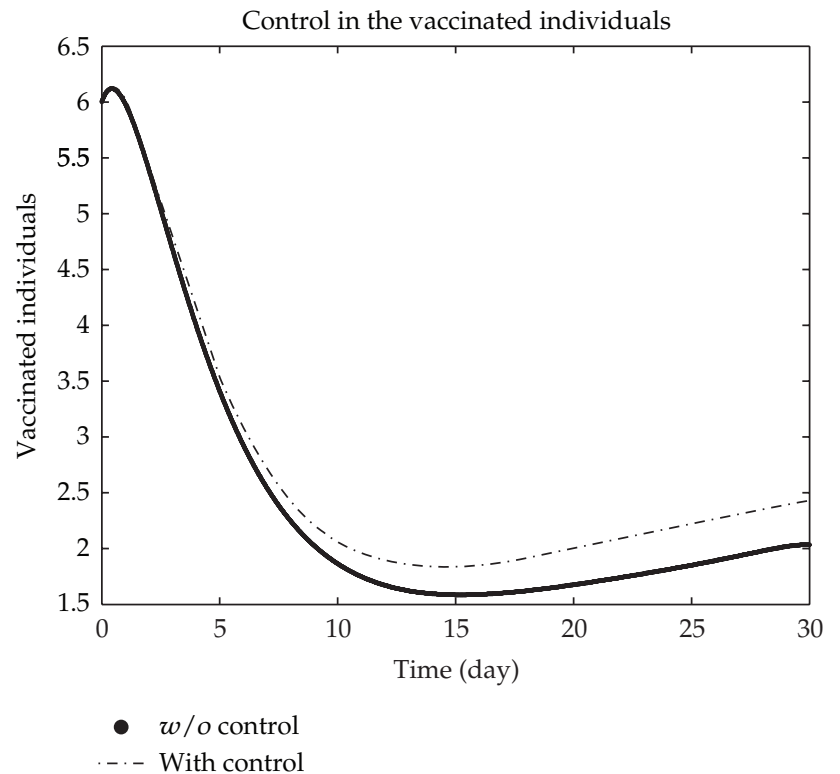

Figure 2: The plot represents the population of vaccinated class with and without control.

Step 3. For $i=1, \ldots, n-1$, write $S^{*}\left(t_{i}\right)=S^{i}, V^{*}\left(t_{i}\right)=V^{i}, E^{*}\left(t_{i}\right)=E^{i}, I^{*}\left(t_{i}\right)=I^{i}, T^{*}\left(t_{i}\right)=$ $T^{i}, R^{*}\left(t_{i}\right)=R^{i}, u_{1}^{*}\left(t_{i}\right)=u_{1}^{i}, u_{2}^{*}\left(t_{i}\right)=u_{2}^{i}, u_{3}^{*}\left(t_{i}\right)=u_{3}^{i}, u_{4}^{*}\left(t_{i}\right)=u_{4}^{i}$ end for.

We have plotted susceptible, vaccinated, exposed, infected, treated, and recovered population with and without control by considering real parameter values given in 


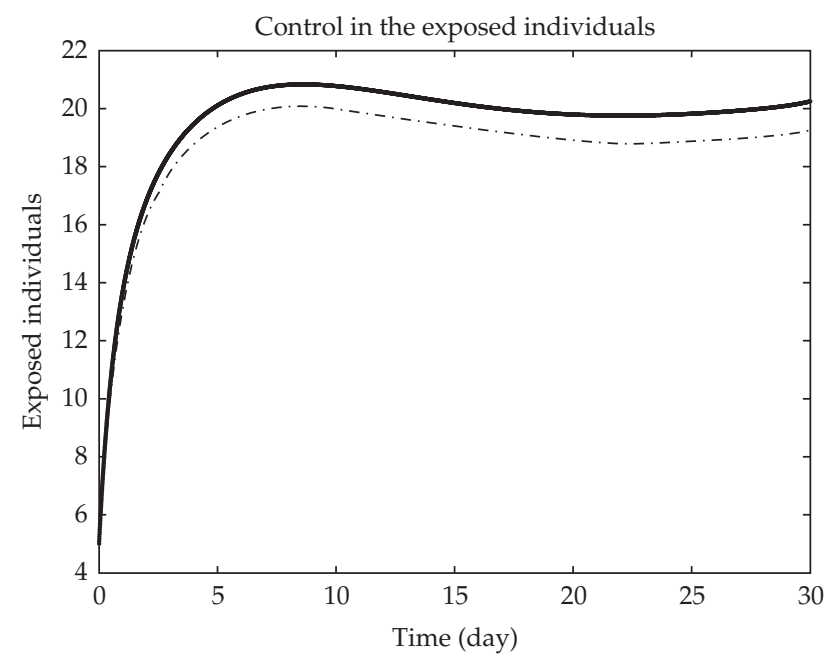

- $w / o$ control

-..- With control

Figure 3: The plot represents the population of exposed class with and without control.

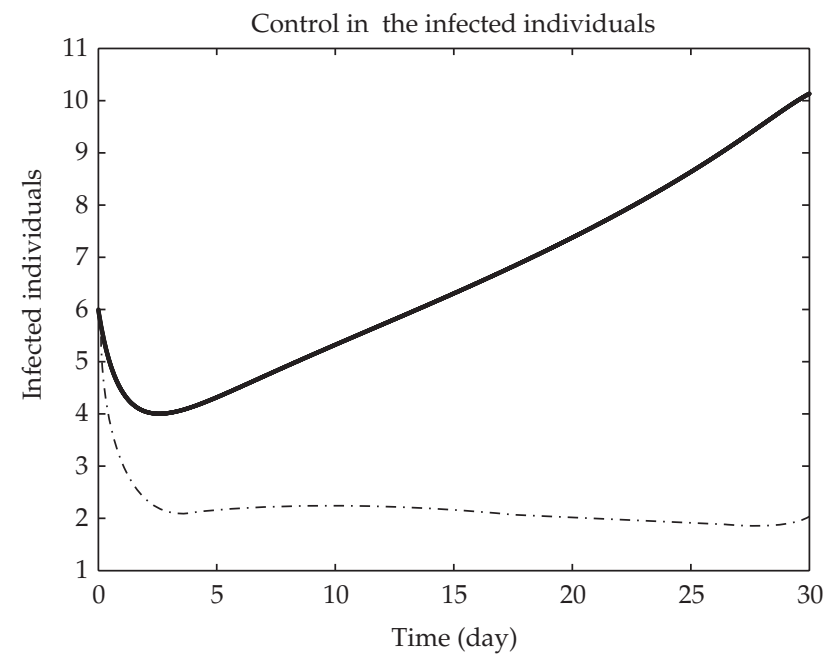

- $w / o$ control

-.. With control

Figure 4: The plot represents the population of infected class with and without control.

Table 1, with initial values $S(0)=15, V(0)=6, E(0)=5, I(0)=6, T(0)=4, R(0)=2$. In each of the given graphes the undashed and the dashed lines represent the individuals without and with control, respectively. The weight constant of the objective functional is $A_{1}=0.05, A_{2}=0.09, c_{1}=0.1, c_{2}=1.1, c_{3}=1.5$, and $c_{4}=0.3$. Figure 1 shows the population of the susceptible individuals with and without control, Figure 2 represents the population of the vaccinated individuals with and without control, and we see that the population of the vaccinated individuals increased after control. Figure 3 represents the population of the 


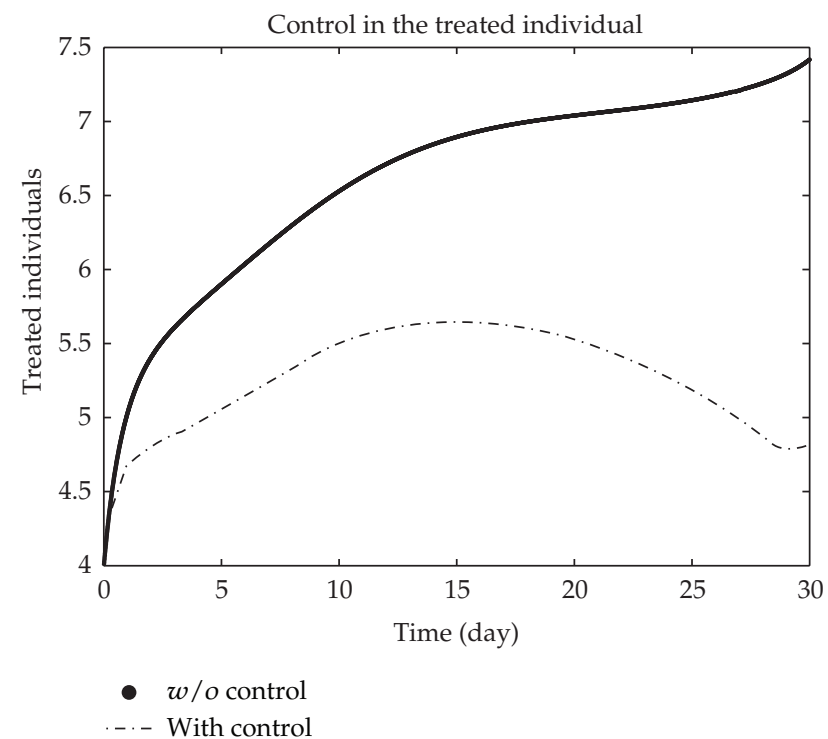

Figure 5: The plot represents the population of treated class with and without control.

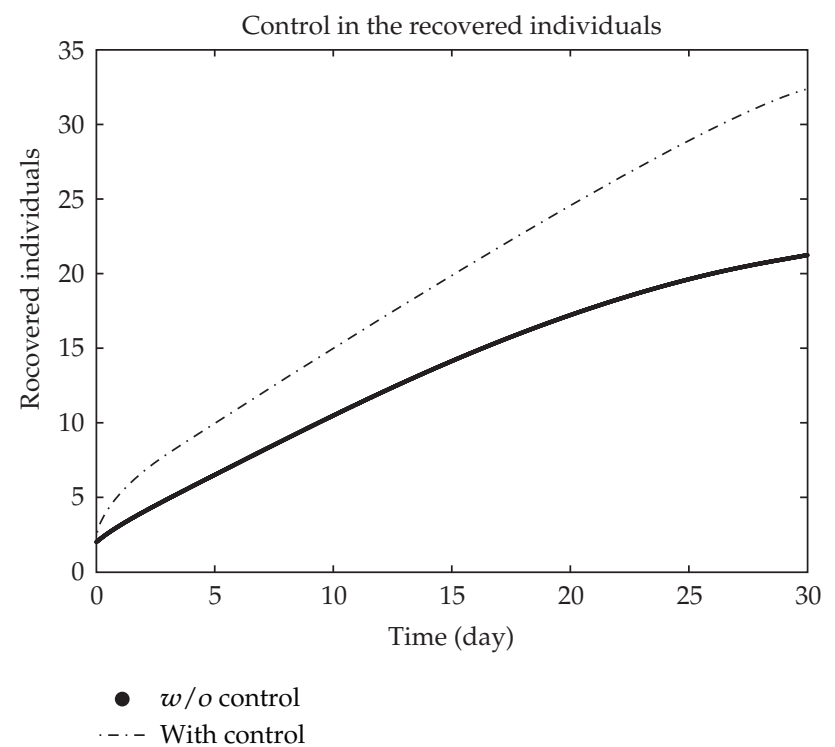

Figure 6: The plot represents the population of recovered class with and without control.

exposed individuals with and without control. In Figure 4 we see that the infected individuals with control decreased more sharply than that of without control. Figure 5 shows that per day clinically reported individuals decreased after control, and in Figure 6 we see that the number of recovered individuals with control increased more sharply than that of without control. Figure 7 shows the plots of control variables. 


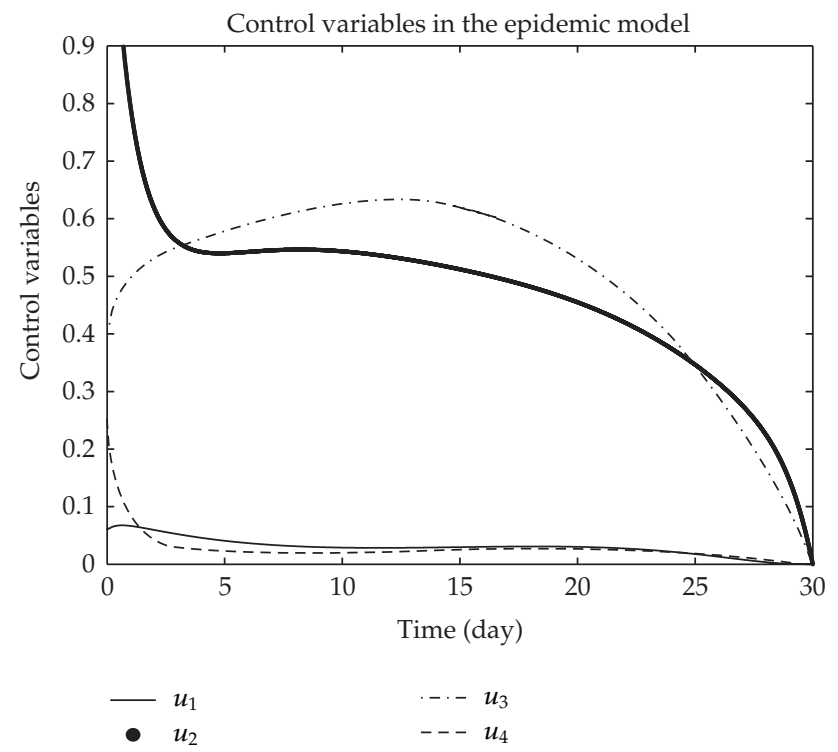

Figure 7: The plot shows control variables $u_{1}, u_{2}, u_{3}, u_{4}$.

Table 1: Parameter values used for numerical simulations.

\begin{tabular}{lcc}
\hline Notation & Parameters definition & Value \\
\hline$\Lambda$ & Recruitment rate & $0.9 /$ day \\
$\beta$ & Recovery rate due to treatment & $0.14 /$ day \\
$\alpha_{1}$ & Effective contact rate between $S$ an $I$ & $0.003 /$ day \\
$\alpha_{2}$ & Effective contact rate between $S$ an $E$ & $0.145 /$ day \\
$\alpha_{3}$ & The rate at which individuals transfer from $E$ to $I$ & $0.07 /$ day \\
$\theta$ & Treatment effectiveness as a reduction factor in disease-induced death & $0.1 /$ day \\
$\epsilon$ & The natural recovery rate of infected individuals & $0.14 /$ day \\
$\mu$ & Natural death rate & $0.0009 /$ day \\
$\nu$ & Disease-induced death rate & 0.002 \\
$r$ & Rate of immunity loss & $0.02 /$ day \\
$\sigma$ & Vaccine efficacy & 0.3 \\
$k$ & Rate at which vaccine wanes & 0.15 \\
$\phi$ & Vaccine uptake rate & $0.35 /$ day \\
$\omega$ & Treatment rate & 0.4 \\
\hline
\end{tabular}

\section{Conclusion}

An optimal control problem of the transmission dynamics of the human influenza disease has been presented. We sought to determine four types of control functions associated with minimizing the wanning of vaccination, isolating the clinically infectious people and antiviral treatment of the clinically infected people and the hospitalized people. Our control plots indicated that the number of exposed, infected, and hospitalized people decreased in the optimal system. We developed the necessary conditions for the existence of the optimal control by using the Pontryagin's Maximum Principle. Using the state and adjoint system together with the characterization of the optimal control, we solved the problem numerically 
by an efficient numerical method based on optimal control with the estimated parameter values based on influenza. The results showed that the control practices are very effective in reducing the incidence of infectious population.

\section{References}

[1] C. J. Murray, A. D. Lopez, B. Chin, D. Feehan, and K. H. Hill, “Estimation of potential global pandemic influenza mortality on the basis of vital registry data from the 1918-1920 pandemic: a quantitative analysis," The Lancet, vol. 368, no. 9554, pp. 2211-2218, 2006.

[2] USGAO Influenza Pandemic, "Efforts underway to address constraints on using antivirals and vaccines to forestall a pandemic," Report Congressional Request, 2007.

[3] N. J. Cox and K. Subbarao, “Influenza," The Lancet, vol. 354, no. 9186, pp. 1277-1282, 1999.

[4] S. F. Regan and C. Fowler, "Influenza. Past, present, and future," Journal of Gerontological Nursing, vol. 28, pp. 30-37, 2002.

[5] World Health Organization, http://www.who.int/mediacentre/factshaats/2003/fs211/en, 2004.

[6] M. E. Alexander, C. Bowman, S. M. Moghadas, R. Summers, A. B. Gumel, and B. M. Sahai, "A vaccination model for transmission dynamics of influenza," SIAM Journal on Applied Dynamical Systems, vol. 3, no. 4, pp. 503-524, 2004.

[7] S. Iwami, Y. Takeuchi, and X. Liu, "Avian-human influenza epidemic model," Mathematical Biosciences, vol. 207, no. 1, pp. 1-25, 2007.

[8] M. Nuño, G. Chowell, and A. B. Gumel, "Assessing the role of basic control measures, antivirals and vaccine in curtailing pandemic influenza: scenarios for the US, UK and the Netherlands," Journal of the Royal Society Interface, vol. 4, no. 14, pp. 505-521, 2007.

[9] S. Lee, G. Chowell, and C. Castillo-Chávez, "Optimal control for pandemic influenza: the role of limited antiviral treatment and isolation," Journal of Theoretical Biology, vol. 265, no. 2, pp. 136-150, 2010.

[10] G. Birkhoff and G.-C. Rota, Ordinary Differential Equations, John Wiley \& Sons Inc., New York, Ny, USA, 4th edition, 1989.

[11] D. L. Lukes, Differential Equations: Classical to Controlled, Mathematics in Science and Engineering, vol. 162, Academic Press, New York, NY, USA, 1982.

[12] G. Zaman, Y. Han Kang, and I. H. Jung, "Stability analysis and optimal vaccination of an SIR epidemic model," BioSystems, vol. 93, no. 3, pp. 240-249, 2008.

[13] A. A. Lashari and G. Zaman, "Optimal control of a vector borne disease with horizontal transmission," Nonlinear Analysis. Real World Applications, vol. 13, no. 1, pp. 203-212, 2012.

[14] M. I. Kamien and N. L. Schwartz, Dynamics Optimization: The Calculus of Variations and Optimal Control in Economics and Management, Elsevier Science, 1991.

[15] A. B. Gumel, P. N. Shivakumar, and B. M. Sahai, "A mathematical model for the dynamics of HIV-1 during the typical course of infection," in Proceedings of the 3rd World Congress of Nonlinear Analysts, vol. 47 , no. 3, pp. 1773-1783. 


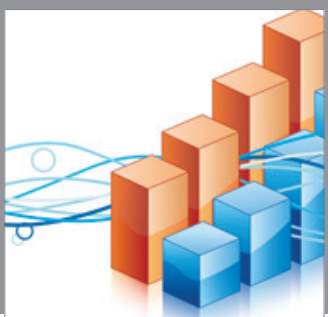

Advances in

Operations Research

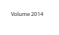

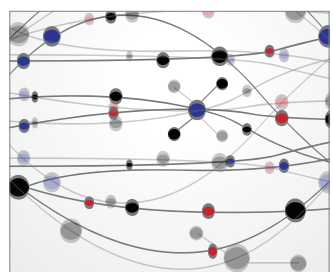

\section{The Scientific} World Journal
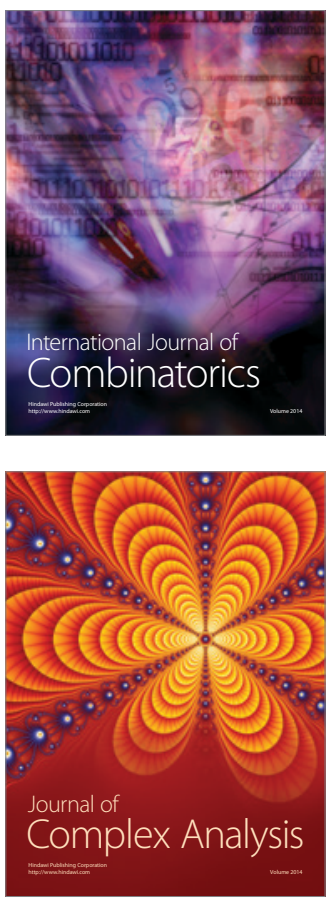

International Journal of

Mathematics and

Mathematical

Sciences
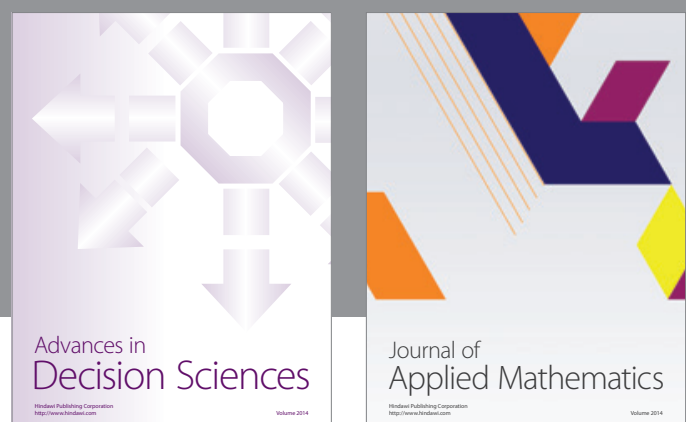

Journal of

Applied Mathematics
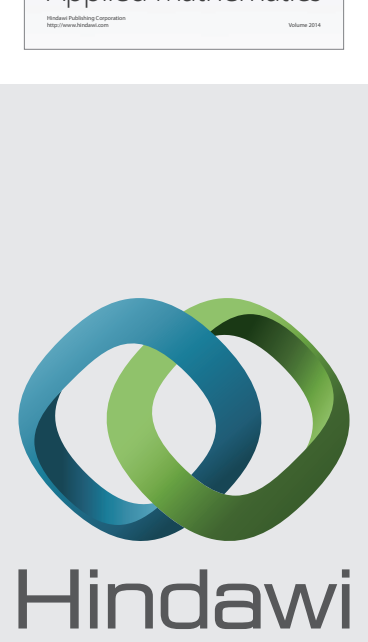

Submit your manuscripts at http://www.hindawi.com
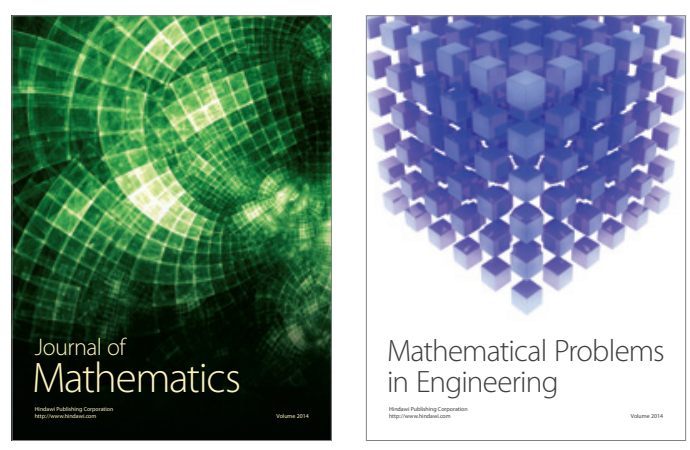

Mathematical Problems in Engineering
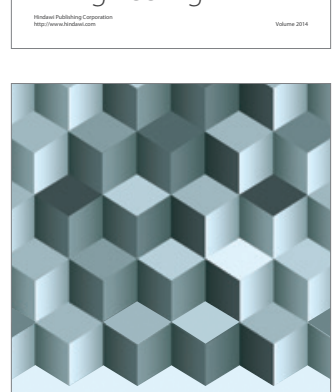

Journal of

Function Spaces
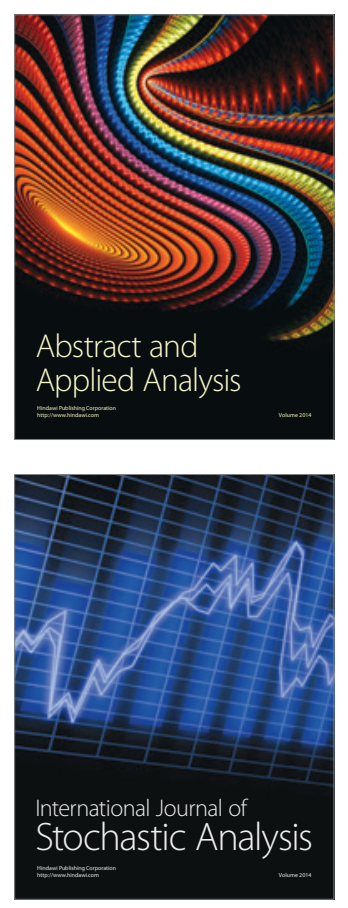

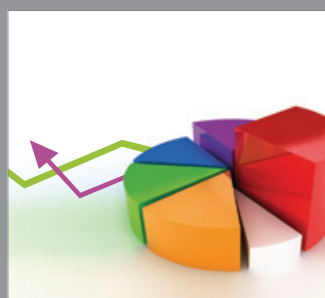

ournal of

Probability and Statistics

Promensencen
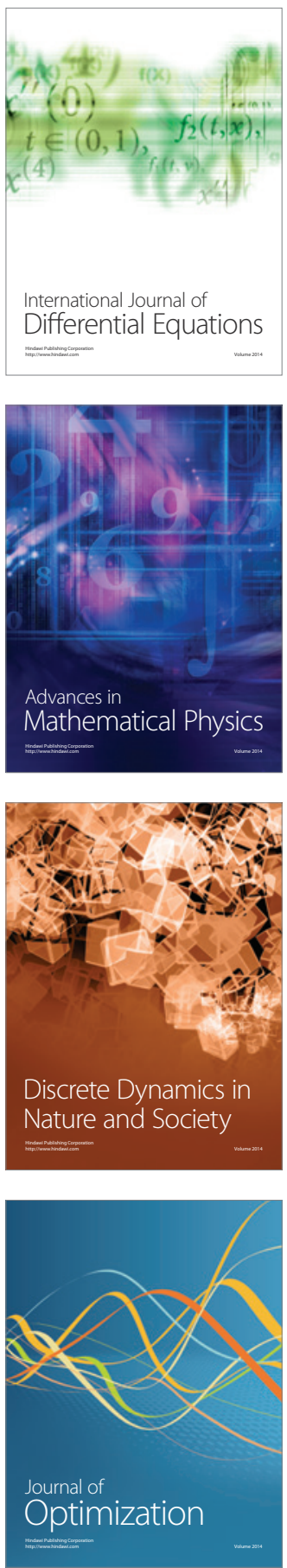